\title{
Studies on the induction of pregnancy and the number of fetuses during pregnancy in rats
}

\author{
Seung-Hee Choi ${ }^{1,3}$, Yong-Seong Cho ${ }^{1}$, Min-Ji Kim ${ }^{1}$, Chae-Hyeok Lee ${ }^{1}$, Hwan-Hoo Seong ${ }^{1}$, \\ Soon-Hwa Baek ${ }^{2}$ and Jang-Hee Lee ${ }^{1, *}$ \\ ${ }^{1}$ Department of Companion Animal, Seoul Hoseo Occupational Training College, Seoul 07583, Korea \\ ${ }^{2}$ Department of ICT, Baekseok University, Cheonan 31065, Korea \\ ${ }^{3}$ Animal Biotechnology, Graduate School of Animal Life Convergence Science, Institute of Genetic Engineering, \\ Hankyong National University, Anseong 17579, Korea
}

Received July 21, 2020

Revised August 31, 2020

Accepted September 1, 2020

\section{*Correspondence}

Jang-Hee Lee

E-mail: bio-culture@hanmail.net

ORCID

https://orcid.org/0000-0003-3998-6519

\begin{abstract}
This study used adult wistar-based rats to observe the sexual cycle as a morphological characteristic of vaginal epithelial cells by vaginal smearing, and investigated the fetal number through mating with male rats of the same strain. The target animal was a 12 to 13-week-old Wistar-based mature unlighted rat (weight $220 \mathrm{~g}$ to $240 \mathrm{~g}$ ), room temperature $23 \pm 2^{\circ} \mathrm{C}, 14$ hours artificial lighting (05:00 to 19:00 hours), 10 hours Adapted individuals were used for rearing for at least 2 weeks under the conditions of the darkroom (19:00 to 05:00). The feed was managed for free feeding of pellet feed for animals and water. The vaginal smearing method was used for the experiments by observing the sexual cycle every morning and confirming that the normal sexual cycle of 4 or 5 days was repeated at least 2 cycles or more. As a result, the proestrus was found to have few red blood cells, the cells and nuclei were rather large and round, and many nucleated cells were identified. In the case of the estrus, the cells were large and the nuclei were not stained, and most of the keratinocytes were found. In addition, in the metestrus and diestrus, there were many white blood cells, and it was confirmed that nucleated epithelial cells and keratinocytes were significantly reduced. The pregnancy period was $21 \pm 1.8$ days, and the number of live births per delivery was 11.9 on average. The number of fetuses on the 8th and 10th days of pregnancy were $15.2 \pm 0.4$ and $15.4 \pm 0.3$, respectively. On the contrary, the number of fetuses on the 12 th day of pregnancy was $12.9 \pm 0.6$, which was significantly $(p<0.05)$ decreased compared to the 10th day of pregnancy, and the number of fetuses was similar until delivery. As a result of investigating the change of body weight according to the birth weight and growth stage after delivery, the birth weight of female and male was $9.2 \pm 2.0 \mathrm{~g}$ and $9.8 \pm 2.5 \mathrm{~g}$, respectively. After that, until the 16th day, the female and the male showed similarly moderate weight gain, and then showed a rapid weight gain until the 21st day of lactation. With reference to the results of this study, it is expected to be used as basic data for determining the mating time of rodents and controlling pregnancy and fetal number.
\end{abstract}

Keywords: delivery, estrus cycle, fetus, keratinocytes, pregnancy, rat 


\section{INTRODUCTION}

In mammals, the female estrus cycle refers to the periodic physiological state of estrogen secretion and $\mathrm{LH}$ surge followed by ovulation from the mature ovary of the ovary (Shiota et al., 1993; Seong et al., 1992). In other words, the estrus cycle in mammals is a physiological phenomenon manifested by the interaction of the hypothalamic-pituitary-ovarian cycle, which is regulated by the environment and endocrine factors (Mahoney et al., 2010).

In the case of rodents, morphological changes such as keratinization occur as the vaginal epithelial cell layer periodically changes due to the effects of estrogens secreted by follicles during the estrus. Vaginal cytology is a method that can confirm the estrus cycle through these cell layer changes (Byers et al., 2012). It is classified into four stages: proestrus, estrus, metestrus, and diestrus, based on the difference in the cell type and ratio of each cell that appears at each stage in the process of repetitive estrus cycle (Akinloye and Oke, 2010; Byers et al., 2012).

In addition, periodic estrus cycles are repeated with estrogen secreted by follicles during estrus and estrus, and progesterone secreted from the ovary luteum during late estrus and estrus (Wallen, 1990; Mahoney et al., 2010).

In addition, ovulated mature follicles are composed of granulosa cells, theca interna cells, and blood components to form a corpus luteum and secrete progesterone from luteal cells (Seong et al., 1992).

In rodents including rats, after ovulation, the newly formed corpus luteum degenerates and the next follicle develops. If stimulated by males, the corpus luteum formed after ovulation does not degenerate, but progesterone is secreted from luteal cells to maintain pregnancy (Seong et al., 1992; Shiota et al., 1993; Seong, 1996).

However, few studies have been conducted to investigate changes in the number of fetuses during pregnancy and finally the number of individuals induced to delivery in preparation for the number of luteal ovaries produced after ovulation.

Therefore, this study aimed at examining the estrus cycle using vaginal smearing in rats, examining the number of fetuses during pregnancy after mating, and finally examining how fetal numbers are changing until delivery. The mating was conducted with males of the same species to change the number of fetuses by pregnancy period.

\section{MATERIALS AND METHODS}

\section{General information}

We used 40 females and 10 males of mature Wisterbased rats, aged 14-16 weeks, with a weight of about 260-280 g in females and about 300-340 g in males. Contrast cycle is adjusted to artificial lighting at 14:10 (Light: 7:00-21:00, Dark: 21:00-7:00), and feed (pellet feed, rat feed) and fresh water are supplied daily without limitation.

\section{Observation of estrus cycle by vaginal smearing}

The division of the estrus cycle by vaginal smear was classified by referring to the Park et al. (2017) method. That is, from 10 to 12 am every day, the subject was observed for vaginal smearing and the normal estrus cycle of 4 days or 5 days was confirmed to be repeated for at least 2 cycles or more and used in the experiment. In the vaginal smearing method, approximately $200 \mu \mathrm{L}$ of physiological saline is injected into the vagina using an eyedropper, vaginal mucus and vaginal epithelial cells are collected, spread on a slide glass, dried on a warm plate of about 40 degrees for about 10-20 minutes, and then washed with 3\% Giemsa solution, washed with water, dried in the same way, and examined with an optical microscope, the size of keratinocytes, endometrial cells, the presence of nuclei and the presence of leukocytes were analyzed. Cycles were separated.

That is, in the proestrus, there are few red blood cells and white blood cells, the cells are rather large and round, and many nucleated cells and some keratinized nucleated cells have been identified. In the case of the estrus, the cell nuclei are not identified. Cells were large and mostly cornified epithelial cells existed, some of which were agglomerated. Also, in the case of metestrus and diestrus, there were a lot of red blood cells and white blood cells, and there were few nucleated epithelial cells and rarely identified keratinocytes (Fig. 1).

Three cell types are identified: large nucleated cornified epithelial cells (white arrow), Large unnucleated cornified epithelial cells (black arrowhead), nucleated epithelial cells (black arrowhead) and leukocytes (circle). Stage of estrus include A: proestrus, B: estrus, C: Metestrus, D: Ditestrus. 

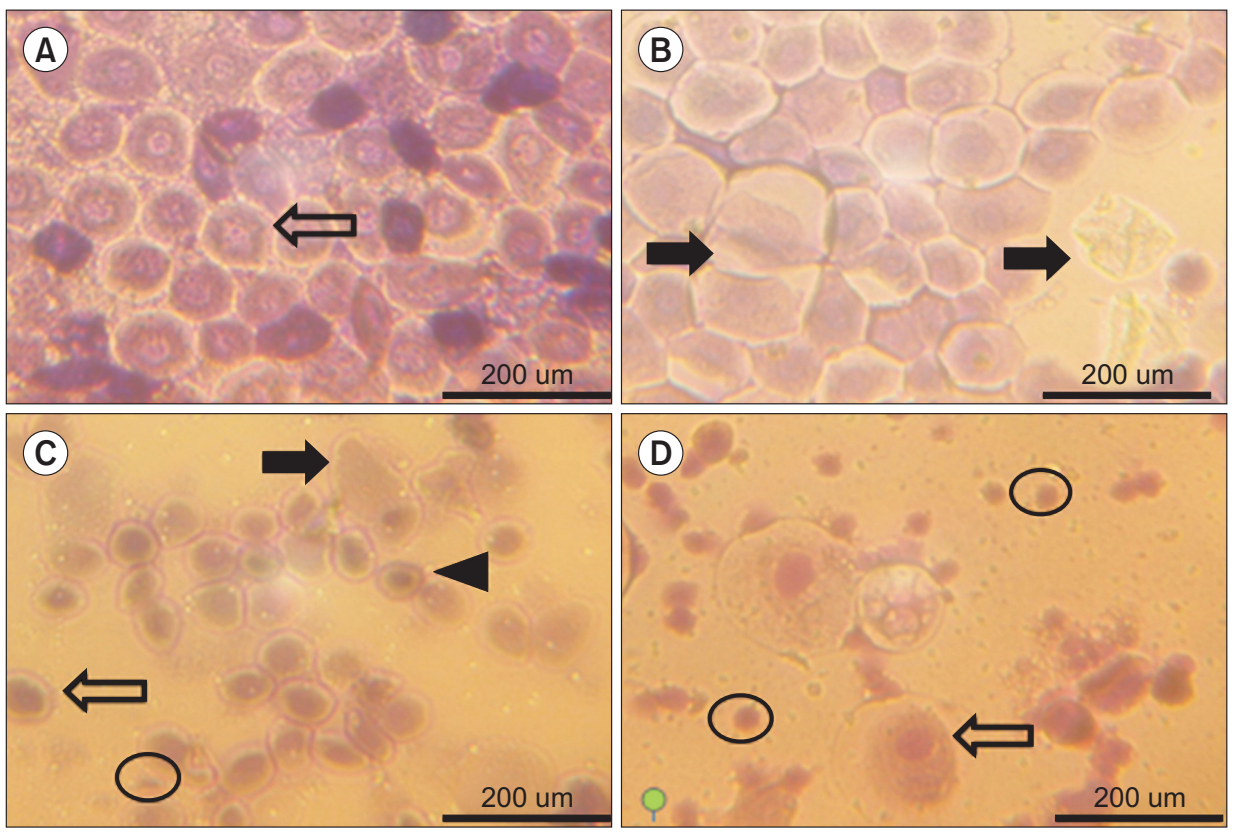

Fig. 1. Each cell types by vaginal cytology representing each stage of estrous in wister rats. (A) Schematic diagram of a small intermediate epithelial cell from the rat vaginal. (B) Schematic diagram of a large intermediate epithelial cell from the rat vaginal. (C) Schematic diagram of a superficial epithelial cell with a nucleus that is showing signs of karyorrhexis (chromatin disintergrates into formless granules, or appears to fade). (D) Schematic diagram of an annucleated superficial epithelial cell.

\section{Induction of pregnancy}

In the afternoon of proestrus, the male and female were combined in a male cage to induce pregnancy. The next morning, an individual whose vaginal sperm was identified was designated as the 0th day of pregnancy.

\section{Changes in the number of fetuses during pregnancy}

On the 8 th and 10th, 12th, 14th, 16th, and 18th days of pregnancy and at the time of delivery (Day 21), 3-4 animals were killed immediately by cervical dislocation. The number of fetuses in the uterus was checked, and some subjects were measured by dividing the weight between male and female at birth and after weaning.

\section{Statistics processing}

The number of fetuses per gestation period was calculated as mean \pm standard error (Mean \pm SEM), and the comparison of the number of fetuses per gestation period was examined for significance using the Statistical Analysis System (SAS).

\section{RESULTS}

\section{Confirmation of estrus cycle by morphological characteristics of vaginal mucus cells}

As shown in Fig. 1, in the proestrus (A), the vaginal membrane cells were large and rounded, and the cell nuclei were confirmed. In the estrus (B), endometrial cells
A

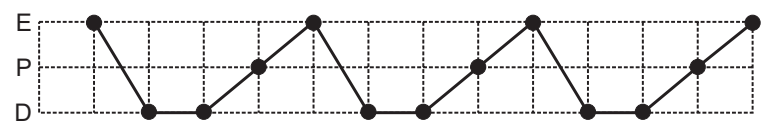

B

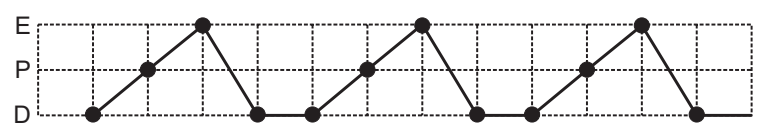

C

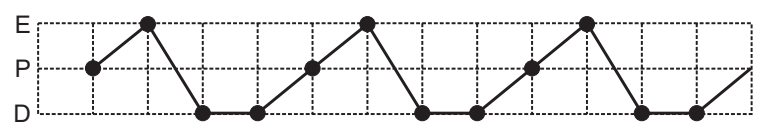

D

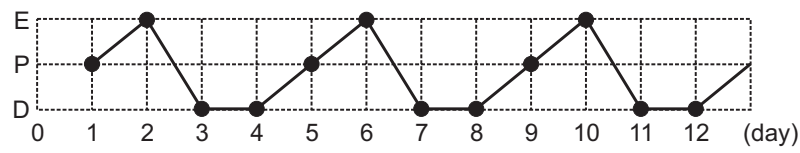

${ }^{*} \mathrm{D}$ : diestrus, E: estrus, P: proestrus

Fig. 2. Changes on vaginal epithelial cells of vaginal smear method in female after mating with the same strain male rats. In this figure A, B, C, D represent the estrus cycle of an individual randomly selected in each estrus cycle stage.

were large and mostly composed of keratinocytes, and the cell nuclei were not stained, and in some cells, the cell nuclei were confirmed to be small. In addition, a lot of mucus secretions could be identified at this time. In addition, the metestrus (C) was generally small in cell size, and was able to identify a large number of white blood cells and red blood cells, and rarely identified keratinocytes. In the diestrus (D), a large number of red blood cells and white blood cells were identified, and the cell nuclei of somewhat smaller vaginal epithelial cells were identified.

Fig. 2 shows the results obtained by collecting and ex- 
amining vaginal epithelial cells every day in the method shown in Fig. 1. As shown in Fig. 2, it was confirmed that a normal 4 days estrus cycle was repeated in a mature unlighted wistar-based rat, and rarely, there were individuals with a 5-day sexual cycle, but statistically significant values could not be identified.

In this experiment, the proestrus and the estrus were found to be able to easily determine the sex cycle by the ratio of keratinocytes and cell nuclei. However, the sexual cycles of the metestrus and the diestrus were difficult to distinguish.

\section{Changes in the number of fetuses during pregnancy}

In order to investigate the change in the number of fetuses during pregnancy, females in the state of estrus were selected by vaginal smearing and mating was performed by merging the same type of individuals into the male cages in the afternoon of the day. As a result of checking the vaginal epithelial cells the next morning, some individuals identified vaginal plugs and sperm. After that, the estrus cycle was examined until delivery to the same method (Fig. 3).

After the estrous stage of the female is confirmed as proestrus and mated with the same male. The next morning, sperm mass was found in vaginal smears (Day 0). After 19 days of pregnancy, vaginal smears revealed a diestrus (Fig. 3).

Table 1 shows the change in the number of fetuses every 2-3 days from the 8th day of pregnancy to the expected delivery day (Day 21). As shown in Table 1 . The number of fetuses on the 8th and 10th day of pregnancy was $15.2 \pm$ 0.4 and $15.4 \pm 0.3$, respectively. In contrast, the number of fetuses on the 12th, 16th, 18th, and 20th days of pregnancy was $12.9 \pm 0.6,12.6 \pm 0.3,12.8 \pm 0.3$, and $12.4 \pm$ 0.6 , respectively, and $11.9 \pm 0.9$ at delivery. As such, the unexpected results in this experiment were significantly $(p<0.05)$ decreased fetal numbers between the 10th and

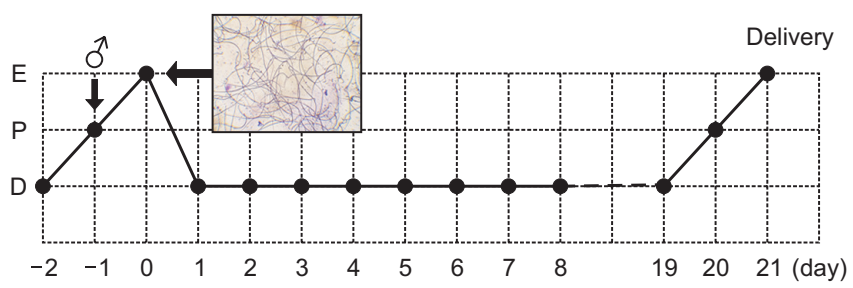

Fig. 3. Changes on vaginal epithelial cells of vaginal smear method in female after mating with the same strain male rats. 12th days of pregnancy, and thereafter, there was no significant difference in fetal number until delivery.

\section{Changes in male and female weight after delivery}

Fig. 4 shows the results of measuring the change in the weight of male and female fetuses by growth stage from birth to 21 days after delivery. The weight at birth immediately after delivery was an average of $9.2 \pm 2.3 \mathrm{~g}$ in

Table 1. Changes in the number of fetuses from early pregnancy to delivery in rats

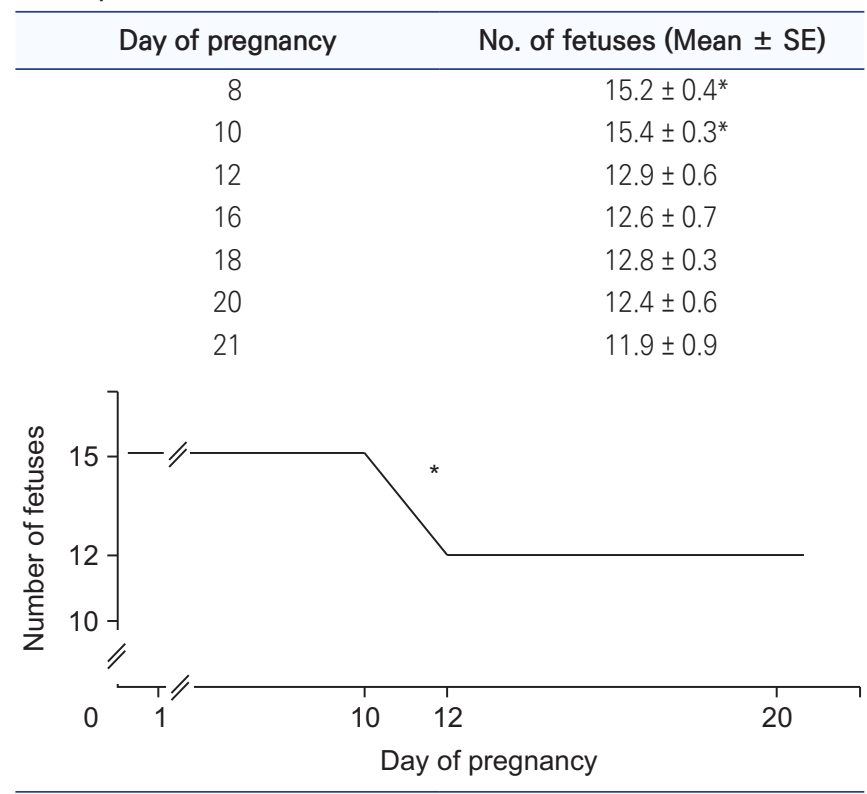

*The number of fetuses on the 8th and 10th day of pregnancy was significantly different from the 12th day of pregnancy to the time of delivery $(p<0.05)$.

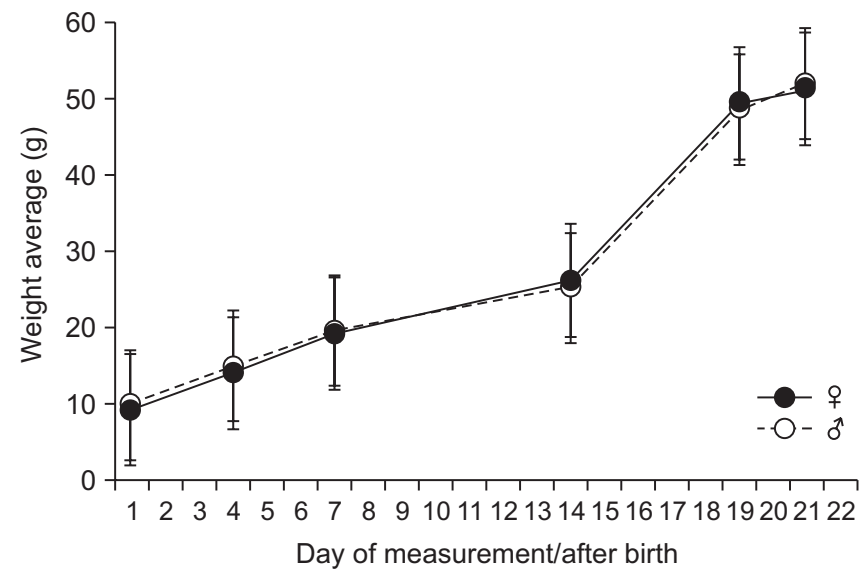

Fig. 4. Changes in male and female fetus weight from postpartum to day 21. There was no significant difference in weight change between males and females on the same day. 
females, but an average of $9.8 \pm 3.7 \mathrm{~g}$ in males. There was no significant difference between sexes. After that, the growth stage showed modest growth until the 14th day after delivery, but significant $(p<0.05)$ growth was observed until the 20th day of the weaning period.

\section{DISCUSSION}

In the results of Fig. 1, the proportion of cells with large and rounded vaginal endometrial cells was high in proestrus and the cell nucleus was stained. In the case of estrus, vaginal endometrial cells were large and consisted mostly of keratinocytes, and the cell nucleus was not stained. In the metestrus and diestrus, red blood cells and white blood cells were generally identified, and vaginal epithelial cells were generally small, but it was confirmed that a large proportion of cells with cell nuclei existed.

Fig. 2 shows the results of daily inspection using the method shown in Fig. 1. It was confirmed that the normal 4-day estrus cycle of mature wistar rats was repeated. Rarely, there were individuals with a 5-day sexual cycle, but statistically significant numbers could not be identified. Therefore, it was found that wistar-type rats under normal feeding control, temperature, and constant light and dark conditions can confirm the correct estrus cycle by vaginal smearing.

Among rodents, in the case of wild animals, there is a difference in cell types and ratios according to the reproductive and non-reproductive seasons, and there is a slight difference according to the time of daylight (Byers et al., 2012).

As a result of using Cricetidae, which mainly lives in Hallasan, Jeju Island, it was reported that the estrous cycle of 4-5 days was regularly repeated depending on the photoperiod (Park et al., 2017). In addition, similar results were reported with mice, rats, and siberian hamster ( $\mathrm{Ph}-$ odopus Sungorous), 4-5 days of estrus cycle (Labyak and Lee, 1995; Jonsson and Silverin, 1997; Marcondes et al., 2002; Jackson and Van Aarde, 2003; Vrooman and Young 2010; Croy et al., 2014).

In addition, in this experiment, the collection time of the vaginal mucosa cells was performed between 9 am and $12 \mathrm{pm}$ every day, and it was generally possible to observe the exact estrus cycle. However, if the sampling time was irregular, there was some difference in determining the correct estrus cycle. Therefore, in order to confirm the correct estrus cycle, it is necessary to collect vaginal epithelial cells at the same time every day with appropriate specification environment.

On the other hand, dogs, which are popular as pets, show estrus every 6 months, have a long estrus cycle, and have various symptoms of estrus for each individual, making it difficult to directly determine the right time to fertilize and the exact time of ovulation (Concannon and Lein, 1989; Allen, 1992). In addition, Park et al. (2013) reported that the day when the ratio of keratinized vaginal epithelial cells in small dogs increased to more than $90 \%$ was able to predict the delivery date at the same time as the time of mating. Therefore, it is judged that the vaginal epithelial cytology method can be used industrially as a means of confirming the time for artificial insemination and insemination of dogs (Allen, 1992; Feldman and Nelson, 1996).

In the future, it is expected that it will be highly utilized for judging the mating time and proliferation of specific pets depending on the vaginal epithelial cytology method.

In order to induce pregnancy, crosses were carried out with males of the same breed. The next morning after mating, pregnancy was confirmed by checking vaginal plugs and sperm cells. In addition, as a result of examining the vaginal smear until delivery by the same method, it was confirmed that it remained in the diestrus state until delivery (Fig. 3).

Therefore, pregnancy maintenance is presumed that progesterone is secreted from the new luteal body by mating stimulation of males and plays a physiological role in protecting the fetus until delivery (Smith et al., 1975; Shiota et al., 1993).

As shown in Table 1. The number of fetuses on the 8 th and 10th day of pregnancy was $15.2 \pm 0.4$ and $15.4 \pm 0.3$, respectively, but the number of fetuses on the 12th, 16th, 18th, and 20th day of pregnancy was respectively. $12.9 \pm$ $0.6,12.6 \pm 0.3,12.8 \pm 0.3,12.4 \pm 0.6$, and $11.9 \pm 0.9$ at delivery. As described above, the unexpected result in this experiment was that the number of fetuses was significantly decreased $(p<0.05)$ between the 10th and 12th days of pregnancy, and thereafter, there was no significant difference in the number of fetuses until delivery.

In rodents such as rats, the corpus luteum formed after ovulation does not secrete progesterone and immediately regresses and repeats a short estrus cycle (4-5 days) in which new follicles develop. This is because the activity of 
$20 \alpha$-Hydroxysteroid dehydrogenase (20 $\alpha$-HSD) in the corpus luteum is high, and this enzyme metabolizes progesterone to biologically inactive $20 \alpha$-dihydroprogesterone (20 $\alpha$-OHP) (Seong et al., 1992, 1995; Shiota et al., 1993).

Therefore, to analyze the significant difference in the number of fetuses between the 10th and 12th days of pregnancy, additional studies on steroid hormones and molecular biology are considered necessary.

Fig. 4 shows the results of measuring the change in the weight of male and female fetuses by growth stage from birth to 21 days after delivery. The weight at birth immediately after delivery was an average of $9.2 \pm 2.3 \mathrm{~g}$ in females, but an average of $9.8 \pm 3.7 \mathrm{~g}$ in males. There was no significant difference between sexes. After that, the growth stage showed modest growth until the 14th day after delivery, but significant $(p<0.05)$ growth was observed until the 20th day of the weaning period.

These results indicate that the individual is growing rapidly by ingesting the mother's pellet feed as the weaning period from the 3 rd week after delivery.

\section{CONFLICTS OF INTEREST}

No potential conflict of interest relevant to this article was reported.

\section{AUTHOR CONTRIBUTIONS}

Conceptulization: Seung-Hee Choi, Yong-Seong Cho, Min-Ji Kim, Chae-Hyeok Lee, Hwan-Hoo Seong, SoonHwa Baek, and Jang-Hee Lee

\section{AUTHOR'S POSITION AND ORCID NO.}

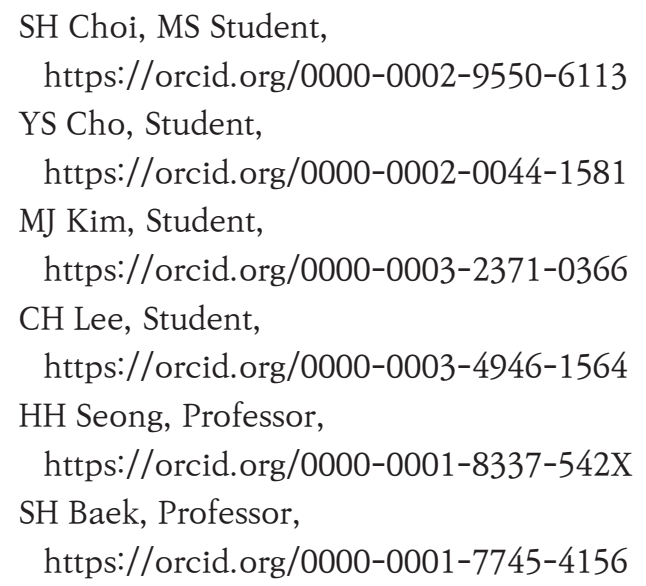

JH Lee, Professor,

https://orcid.org/0000-0003-3998-6519

\section{REFERENCES}

Akinloye AK and Oke BO. 2010. Characterization of the uterus and mammary glands of the female African giant rats (Cricetomys gambianus, Waterhouse) in Nigeria. Int. J. Morphol. 28:93-96.

Allen WE. 1992. Physiology of the oestrous cycle. In: Allen WE (Ed.), Fertility and Obstetrics in the Dog. Blackwell Scientific Publications, Oxford, pp. 13-90.

Byers SL, Wiles MV, Dunn SL, Taft RA. 2012. Mouse estrous cycle identification tool and images. PLoS One 7:e35538.

Concannon PW and Lein DH. 1989. Hormonal and clinical correlates of ovarian cycles, ovulation, pseudopregnancy and pregnancy in dogs. In: Kirk RW (Ed.), Current Veterinary Therapy. 10th ed, Saunders, Philadelphia, pp. 1269-1282.

Croy BA, Yamada AT, DeMayo FJ, Adamson SL. 2014. The guide to investigation of mouse pregnancy. Academic Press, London, pp. 3-19.

Feldman EC and Nelson RW. 1996. The ovarian cycle and vaginal cytology. In: Feldman EC and Nelson RW (Eds.), Canine and Feline Endocrinology and Reproduction. 2nd ed, Saunders, Philadelphia, pp. 526-546.

Jackson TP and Van Aarde RJ. 2003. Sex- and species-specific growth patterns in cryptic African rodents, Mastomys natalensis and M. coucha. J. Mammal. 84:851-860.

Jonsson P and Silverin B. 1997. The estrous cycle in female wood mice (Apodemus sylvaticus) and the influence of the male. Ann. Zool. Fenn. 34:197-204.

Labyak SE and Lee TM. 1995. Estrus- and steroid-induced changes in circadian rhythms in a diurnal rodent, Octodon degus. Physiol. Behav. 58:573-585.

Mahoney MM, Rossi BV, Hagenauer MH, Lee TM. 2010. Characterization of the estrous cycle in Octodon degus. Biol. Reprod. 84:664-671.

Marcondes FK, Bianchi FJ, Tanno AP. 2002. Determination of the estrous cycle phases of rats: some helpful considerations. Braz. J. Biol. 62(4A):609-614.

Park CH, Yang JY, Park JT, Lee SH, Park IC, Kim JT, Suh GH, Oh KS, Son CH. 2013. Prediction of parturition date based on vaginal cytology in small dogs. J. Emb. Trans. 28:25-30.

Park JH, Ahn KJ, Oh HS. 2017. Estrus cycles of the female Tscherskia triton (Mammalia: Rodentia: Cricetidae) according to the photoperiod. Korean J. Environ. Biol. 35:160-168.

Seong HH. 1996. The endocrine control of corpus luteum function. Korean J. Anim. Reprod. 19:307-322.

Seong HH, Shiota K, Noda K, Ogura A, Asano T, Takahashi M. 1992. Expression of activities of two 20 alpha-hydroxysteroid dehydrogenase isozymes in rat corpora lutea. J. Reprod. Fertil. 96:573-580.

Shiota K, Seong HH, Noda K, Hattori N, Ikeda A, Ogura A, Itagaki S, Takahashi M, Ogawa T. 1993. 20 alpha-Hydroxys- 
teroid dehydrogenase activity in rat placenta. Endocr. J. 40:673-681.

Smith MS, Freeman ME, Neill JD. 1975. The control of progesterone secretion during the estrous cycle and early pseudopregnancy in the rat: prolactin, gonadotropin and steroid levels associated with rescue of the corpus luteum of pseudopregnancy. Endocrinology 96:219-226.

Vrooman LA and Young KA. 2010. Ovarian matrix metallopro- teinases are differentially regulated during the estrous cycle but not during short photoperiod induced regression in Siberian hamsters (Phodopus sungorus). Reprod. Biol. Endocrinol. 8:79.

Wallen K. 1990. Desire and ability: hormones and the regulation of female sexual behavior. Neurosci. Biobehav. Rev. 14:233-241. 